\title{
Learning, Host Fidelity, and the Stability of Host-Parasitoid Communities
}

\author{
Alan Hastings ${ }^{1, \star}$ and H. C. J. Godfray ${ }^{2, \dagger}$ \\ 1. Department of Environmental Science and Policy, Institute of \\ Theoretical Dynamics and Center for Population Biology, \\ University of California, Davis, California 95616; \\ 2. Department of Biology and Natural Environment Research \\ Council, Centre for Population Biology, Imperial College at \\ Silwood Park, Ascot, Berkshire SL5 7PY, United Kingdom \\ Submitted April 9, 1998; Accepted September 30, 1998
}

\begin{abstract}
Simple models of host-parasitoid interactions show that a polyphagous parasitoid tends to drive extinct all but the most fecund of its hosts. Coexistence requires a mechanism by which hosts with lower fecundity can increase when rare. We explore how recently discovered parasitoid learning behavior can contribute to species coexistence. On emergence as adults, parasitoids learn chemical cues associated with their natal host and hence are more likely to locate and to oviposit in hosts of the same species. For two models incorporating different assumptions about parasitoid searching, we derive conditions for the strength of host fidelity that permits a feasible multihost equilibrium and argue that this equilibrium is stable whenever the corresponding single-host equilibria are stable.
\end{abstract}

Keywords: host-parasitoid dynamics, apparent competition, coexistence, parasitoids.

Parasitoid insects are exceedingly abundant members of most terrestrial communities, in terms of both numbers of individuals and numbers of species (Godfray 1994). Their larvae develop by feeding on the bodies of other insects, their hosts, which provide all the resources required for growth to maturity. Parasitoids are often important in regulating the abundances of their hosts (Hassell 1978, 1998; Godfray and Müller 1999), and they have been widely used in biological control against economically important pests (Mills and Getz 1996; Murdoch and Briggs 1996).

* E-mail: amhastings@ucdavis.edu.

† E-mail: c.godfray@ic.ac.uk.

Am. Nat. 1999. Vol. 153, pp. 295-301. (c) 1999 by The University of Chicago. 0003-0147/99/5303-0005\$03.00. All rights reserved.
The majority of parasitoid species attack more than one species of hosts, and food webs that show the trophic relationships in natural communities are typically very complex (Memmott et al. 1994). Despite this widespread pattern, the simplest model of one parasitoid attacking two (or more) hosts predicts that all but one host will go extinct, except in the limiting case of species with identical rates of reproduction (Holt and Lawton 1993). The host that survives is the species with the highest fecundity (say, $\left.\lambda_{\max }\right)$. At equilibrium, the density of parasitoids must be sufficient to reduce the host's potential growth rate from $\lambda_{\max }$ to 1 , which, if all hosts suffer similar risks of parasitism, is enough to drive the growth of all other hosts with $\lambda<\lambda_{\max }$ to below 1 . This mechanism of competitive exclusion is structurally similar to that in many other ecological models that include resource dynamics. For example, in plant competition for nutrients, the species that is able to survive at the lowest equilibrium nutrient level $\left(R^{*}\right)$ is the competitive dominant (Tilman 1982).

Parasitoids, however, do attack more than one host in the field, and several mechanisms have been suggested to permit this. All share the feature of allowing both species, or at least the species with the lower fecundity, to be at an advantage when rare in comparison with the more abundant species in the community. The simplest mechanism is to assume that low-fecundity hosts have a refuge from parasitism (Holt and Lawton 1993). This refuge may be physical and be interpretable as a niche difference or it may be probabilistic: in any generation some host patches are not found by parasitoids and, as long as different species occupy different patches, then a low-fecundity species will have some protection when rare. Another simple mechanism involves parasitoid behavior: if parasitoids switch preferentially to attacking the most abundant species, then rare species are relatively advantaged and coexistence is more likely (Murdoch and Oaten 1975).

The behavioral mechanisms underlying switching have normally been thought to involve aggregation to areas of high host density. In this article, we wish to explore the dynamic consequences of a type of parasitoid behavior 
that gives rise to patterns akin to switching and that has only relatively recently been described. It had once been supposed (Thorpe and Jones 1937) that parasitoids that had developed in one species of host learned as larvae the characteristics of that species, which they then preferentially attacked when they became adult (called "Hopkins's host selection principle"). More careful study has shown that, while development in one host species does predispose the parasitoid to search for it as an adult, the learning does not occur at the larval stage but immediately upon adult emergence. After emergence, the adult parasitoid carefully samples the volatile chemicals in its immediate surroundings and uses them as cues (kairomones) to direct its searching (Vet 1983; Corbet 1985; Vet and Groenewold 1990; Kester and Barbosa 1991; Turlings et al. 1992; Cortesero and Monge 1994). The pupation site is often in a microhabitat characteristic of a particular host species (e.g., on a particular host plant), or the husk of the exhausted host may be present, or host remains may contaminate the pupal cocoon. In all these cases, the parasitoids obtain useful information about the chemical stimuli that are likely to be most useful in host location. However, the preference for the natal host is not absolute and will decay in time unless reinforced by host discovery. The population consequences we seek to explore are the extent to which host fidelity influences population dynamics and contributes to species coexistence.

Host-parasitoid population dynamics can be modeled using difference equations that assume separate and synchronized generations of hosts and parasitoids (Nicholson and Bailey 1935; Hassell 1978) or through systems of differential equations assuming overlapping generations (Nisbet and Gurney 1983). The former models are the simpler to analyze and have been extensively studied, and it is this approach we adopt here.

\section{Model}

We begin with the basic Nicholson-Bailey framework for a one-host, one-parasitoid model. Letting $H(t)$ be the number of hosts in generation $t, P(t)$ be the number of parasitoids in generation $t, \lambda$ be the intrinsic rate of increase for the host, and $\mathrm{f}(P(t))$ be the probability that a host escapes parasitism in generation $t$, we get the model

$$
\begin{aligned}
& H(t+1)=\lambda H(t) \mathrm{f}(P(t)), \\
& P(t+1)=H(t)[1-\mathrm{f}(P(t))] .
\end{aligned}
$$

At this point, we do not need to specify the functional form $\mathrm{f}$, although we will do so below to look at questions of stability.
The extension of this model to a generalist parasitoid and several host species is not quite straightforward, as one needs to be careful in the definition of the probability that a host escapes parasitism. We assume that the probability of each host species escaping parasitism depends on $q P$, where $q$ is a measure of the degree to which searching on a particular host species is mutually exclusive. Thus, if $n$ host species occurred in separate locations, each of which attracted an equal number of parasitoids, we would have $q=1 / n$, while, if all host species occurred together, $q=1$. Below, we will allow the probability of escaping parasitism to depend on the individual host species. We use subscripts to indicate species of hosts, so $H_{i}(t)$ is the number of host $i$ in generation $t$ with corresponding rate of increase $\lambda_{i}$. The model

$$
\begin{aligned}
H_{i}(t+1) & =\lambda_{i} H_{i}(t) \mathrm{f}(q P(t)), \\
P(t+1) & =\left[\sum_{j=1}^{n} H_{j}(t)\right][1-\mathrm{f}(q P(t))]
\end{aligned}
$$

has been used to demonstrate the, at first sight, counterintuitive result that all but one of the hosts the one with the largest $\lambda_{i}$, is eliminated, unless two or more hosts have exactly the same rate of increase, $\lambda_{i}$. (Note that by nondimensionalizing, letting $\tilde{P}=q P$ and $\tilde{H}_{j}=q H_{j}$, our somewhat more general model, eqq. [3] and [4], immediately reduces to the classical form, eqq. [1] and [2]. So the dynamical behavior is unchanged by the addition of the extra parameter q.)

We now modify this model, incorporating the idea that the species of host that a parasitoid emerges from affects its likelihood of parasitizing a particular species of host. We will focus on two alternative models incorporating this general concept, which lead to similar outcomes. In model 1 , each female parasitoid only searches for a single host, and the probability of searching on host $j$ depends on the host the parasitoid emerges from. In model 2, each female parasitoid searches all host species but with different efficiencies, depending on the host the parasitoid emerged from. We will describe and analyze a model for each case.

Model 1

The first possibility we focus on assumes that a parasitoid that emerges from host $j$ may be more likely to lay its eggs on host $j$. Thus, we let $b_{i j}$, with $\sum_{i=1}^{n} b_{i j}=1$ be the probability that a parasitoid emerging from host $j$ in the current generation will lay its eggs on host $i$ in the next generation. We also can assume that $b_{i i}>1 / n$, reflecting the fact that a parasitoid emerging from host $i$ is more likely to lay its eggs on host $i$. We let the number of parasitoids laying 
eggs on host $i$ in generation $t$ be given by $P_{i}(t)$. The resulting model is

$$
\begin{aligned}
H_{i}(t+1) & =\lambda_{i} H_{i}(t) \mathrm{f}\left(P_{i}(t)\right), \\
P_{i}(t+1) & =\sum_{j=1}^{n} b_{i j} H_{j}(t)\left[1-\mathrm{f}\left(P_{j}(t)\right)\right],
\end{aligned}
$$

which we analyze below.

Before we begin our analysis, we first show that the model of (5) and (6) reduces to equations (3) and (4) if the parasitoid searches all hosts species independently, if $b_{i j}=1 / n$ for all $i$ and $j$. In this case,

$$
P_{i}(t+1)=(1 / n) \sum_{j=1}^{n} H_{j}(t)\left[1-\mathrm{f}\left(P_{j}(t)\right)\right],
$$

so $P_{i}(t+1)$ does not depend on $i$. We now define the total parasitoid population to be

$$
P(t)=\sum_{i=1}^{n} P_{i}(t)
$$

In this case, since $P_{i}(t)$ does not depend on $i$, the total parasitoid population is

$$
P(t)=n P_{i}(t)
$$

Thus, after one generation, the equation describing the parasitoid dynamics becomes

$$
P(t+1)=\left[\sum_{j=1}^{n} H_{j}(t)\right][1-\mathrm{f}(P(t) / n)],
$$

which is identical to equation (4), and the equation describing the dynamics of the host species becomes identical to equation (3).

\section{Model 2}

We now develop the model describing the case where all parasitoids search all host species, but with efficiencies depending on the host the parasitoid emerges from. Define $a_{i j}$ to be the relative efficiency of a parasitoid emerging from host $j$ attacking host $i$. We will assume that $a_{j j}>$ $\max _{i} a_{i j}$, meaning that a parasitoid emerging from host $j$ is most efficient when searching on host $j$. In this model, $P_{i}$ is the number of parasitoids emerging from host species $i$. The resulting model is

$$
\begin{aligned}
& H_{i}(t+1)=\lambda_{i} H_{i}(t) \mathrm{f}\left(\sum_{j=1}^{n} a_{i j} P_{j}\right), \\
& P_{i}(t+1)=H_{i}(t)\left[1-\mathrm{f}\left(\sum_{j=1}^{n} a_{i j} P_{j}\right)\right],
\end{aligned}
$$

which we analyze below. Once again it is easy to see that the model (11) and (12) reduces to (3) and (4) if the parasitoid searches all hosts species independently, if $a_{i j}=q$ for all $i, j$.

\section{Analysis}

Although one can analyze the models (5) and (6) and (11) and (12) for an arbitrary number of host species, we present here the case of two hosts, which includes the important ideas and is much easier to understand. We will begin by determining the equilibrium, demonstrating that an equilibrium with both hosts at positive frequencies is possible even if the host growth rates $\lambda_{j}$ differ. We treat the two models separately. We will then focus on a specific form for the function $\mathrm{f}$, both to illustrate the conditions for coexistence and to aid in the consideration of the stability of the equilibrium.

\section{Model 1}

We denote the equilibrium values of the host and parasitoid by $H_{i}^{*}$ and $P_{i}^{*}$. We first note that equation (6) implies that

$$
1=\lambda_{i} \mathrm{f}\left(P_{i}^{*}\right)
$$

so the equilibrium parasitoid density on each host species is simply

$$
P_{i}^{*}=\mathrm{f}^{-1}\left(1 / \lambda_{i}\right)
$$

The model equation (6), after substitution of the equilibrium densities of the two hosts (14) in the parasitism function, yields, after simplification,

$$
\left(\begin{array}{ll}
b_{11}\left(1-1 / \lambda_{1}\right) & b_{12}\left(1-1 / \lambda_{2}\right) \\
b_{21}\left(1-1 / \lambda_{1}\right) & b_{22}\left(1-1 / \lambda_{2}\right)
\end{array}\right)\left(\begin{array}{c}
H_{1}^{*} \\
H_{2}^{*}
\end{array}\right)=\left(\begin{array}{c}
P_{1}^{*} \\
P_{2}^{*}
\end{array}\right) .
$$


Solving for the host equilibria, we obtain

$$
\left(\begin{array}{c}
H_{1}^{*} \\
H_{2}^{*}
\end{array}\right)=\left(\begin{array}{cc}
\frac{b_{22}}{\left(1-\frac{1}{\lambda_{1}}\right) \operatorname{det}(\mathbf{B})} & -\frac{b_{12}}{\left(1-\frac{1}{\lambda_{1}}\right) \operatorname{det}(\mathbf{B})} \\
-\frac{b_{21}}{\left(1-\frac{1}{\lambda_{2}}\right) \operatorname{det}(\mathbf{B})} & \frac{b_{11}}{\left(1-\frac{1}{\lambda_{2}}\right) \operatorname{det}(\mathbf{B})}
\end{array}\right)\left(\begin{array}{l}
P_{1}^{*} \\
P_{2}^{*}
\end{array}\right),
$$

where $\mathbf{B}$ is the matrix with entries $b_{i j}$. Writing this last equation without using the matrix notation, with $j \neq i$, we see that

$$
H_{i}^{*}=\frac{b_{j j} P_{i}^{*}-b_{i j} P_{j}^{*}}{\left(1-1 / \lambda_{i}\right) \operatorname{det}(\mathbf{B})} .
$$

Now note that our assumptions imply $\operatorname{det}(\mathbf{B})>0$, and we can assume that $\lambda_{i}>1$, so the denominator of (17) is positive. We now seek to determine when the numerator of (17) is positive for both species. We first focus on the species with the larger growth rate. We see that if

$$
\lambda_{1}<\lambda_{2}
$$

then

$$
P_{1}^{*}<P_{2}^{*}
$$

and as long as

$$
b_{11} \geq 1 / 2 \geq b_{21}
$$

which we have assumed, then

$$
H_{2}^{*}>0
$$

This is basically the mechanism by which the host with the higher rate of increase eliminates the other host if there is no "preference" by the parasitoid. However, in our model, the host with the lower rate of increase will also have a positive equilibrium frequency, the numerator of (17) will be positive, if

$$
b_{22} P_{1}^{*}-b_{12} P_{2}^{*}>0 \text {. }
$$

We rewrite this condition for maintenance of the species with the lower intrinsic rate of increase as

$$
\frac{b_{22}}{b_{12}}>\frac{P_{2}^{*}}{P_{1}^{*}}=\frac{\mathrm{f}^{-1}\left(1 / \lambda_{2}\right)}{\mathrm{f}^{-1}\left(1 / \lambda_{1}\right)},
$$

which will be satisfied if $b_{22}$, the fidelity of the parasitoid to the more fecund host, is high enough compared to $b_{12}$, the probability of the parasitoid switching from the more fecund host to the less fecund host, relative to the differences in the fecundities of the hosts, as measured by the sizes of the parasitoid populations each would support in isolation. Note, perhaps quite surprisingly, that the degree of fidelity to the less fecund host does not enter into the conditions for coexistence. This is the major result of our article.

\section{Model 2}

We again determine the equilibria of the model. From equation (11) we obtain the system of $n$ equations:

$$
\sum_{j=1}^{n} a_{i j} P_{j}^{*}=\mathrm{f}^{-1}\left(1 / \lambda_{i}\right) .
$$

From equation (12), using (24), we obtain

$$
H_{i}^{*}=\frac{P_{i}^{*}}{1-1 / \lambda_{i}} .
$$

Solutions of (25) will be feasible (all host species positive) if all the $P_{i}^{*}$ are positive and all $\lambda_{i}>1$.

Returning to the equation for the parasitoid equilibrium, we write out the equation for the case of two host species as

$$
\left(\begin{array}{ll}
a_{11} & a_{12} \\
a_{21} & a_{22}
\end{array}\right)\left(\begin{array}{l}
P_{1}^{*} \\
P_{2}^{*}
\end{array}\right)=\left(\begin{array}{l}
\mathrm{f}^{-1}\left(1 / \lambda_{1}\right) \\
\mathrm{f}^{-1}\left(1 / \lambda_{2}\right)
\end{array}\right) .
$$

Solving for the parasitoid equilibria, we obtain

$$
\left(\begin{array}{l}
P_{1}^{*} \\
P_{2}^{*}
\end{array}\right)=\left(\begin{array}{cc}
\frac{a_{22}}{\operatorname{det}(\mathbf{A})} & -\frac{a_{12}}{\operatorname{det}(\mathbf{A})} \\
-\frac{a_{21}}{\operatorname{det}(\mathbf{A})} & \frac{a_{11}}{\operatorname{det}(\mathbf{A})}
\end{array}\right)\left(\begin{array}{c}
\mathrm{f}^{-1}\left(1 / \lambda_{1}\right) \\
\mathrm{f}^{-1}\left(1 / \lambda_{2}\right)
\end{array}\right)
$$

where $A$ is the matrix with entries $a_{i j}$. Writing this last equation without using the matrix notation, with $j \neq i$, we see that

$$
P_{i}^{*}=\frac{a_{j j} \mathrm{f}^{-1}\left(1 / \lambda_{i}\right)-a_{i j} \mathrm{f}^{-1}\left(1 / \lambda_{j}\right)}{\operatorname{det}(\mathbf{A})} .
$$

Again, note that our assumptions imply $\operatorname{det}(\mathbf{A})>0$.

As in our analysis of model 1, we ask whether the numerator of (28) is positive for both species, beginning with the species with the higher growth rate. If we assume that $\lambda_{1}<\lambda_{2}$, then, since $\mathrm{f}$ is a decreasing function, we conclude that 


$$
\mathrm{f}^{-1}\left(1 / \lambda_{2}\right)>\mathrm{f}^{-1}\left(1 / \lambda_{1}\right)
$$

so using $a_{11} \geq a_{21}$, we see that

$$
a_{11} \mathrm{f}^{-1}\left(1 / \lambda_{2}\right)-a_{21} \mathrm{f}^{-1}\left(1 / \lambda_{1}\right)>0,
$$

which implies $P_{2}>0$. Once again, the condition for $P_{1}>0$ takes an essentially identical form, which we write as

$$
\frac{a_{22}}{a_{12}}>\frac{\mathrm{f}^{-1}\left(1 / \lambda_{2}\right)}{\mathrm{f}^{-1}\left(1 / \lambda_{1}\right)}
$$

This condition will be satisfied if $a_{22}$ is large enough relative to $a_{12}$. Finally, as we noted above, if both $P_{1}^{*}$ and $P_{2}^{*}$ are positive, then both $H_{1}^{*}$ and $H_{2}^{*}$ are positive.

Thus, we see that the the mechanism we suggest for allowing the persistence of generalist parasitoids and hosts works with either of the two biological scenarios we have described.

\section{The Negative Binomial}

Our analysis to this point has the advantage of generality, but since we have not specified the functional form for the probability of escape from parasitism, we cannot obtain a numerical estimate of the strength of the mechanism we have proposed that will be required for coexistence of the two host species, nor will we be able to look at stability. Thus, we now turn to a specific functional form, choosing the negative binomial model

$$
\mathrm{f}(P)=(1+\alpha P / k)^{-k},
$$

which yields

$$
P^{*}=\mathrm{f}^{-1}\left(1 / \lambda_{i}\right)=(k / a)\left(\lambda^{(1 / k)}-1\right) .
$$

The negative binomial model is used in two ways to represent parasitoid searching in population dynamic models. First, if hosts are found in patches in the environment, and if parasitoids are $\gamma$ distributed across these patches (independent of host density), within which they search randomly, then the negative binomial is an exact description of the interaction (Chesson and Murdoch 1986). Second, the negative binomial is used as a phenomenological description of an interaction in which the risks of parasitism experienced by hosts increases with parasitoid density but with a decreasing slope (May 1978). In other words, there is a density-dependent reduction in parasitoid per capita efficiency. In a simple one-host, one-parasitoid Nicholson-Bailey model incorporating the negative binomial, the stability criterion is simply $k<1$.
The use of the negative binomial model in the conditions for coexistence, (23) or (31), indicates that coexistence in model 1 requires

$$
\frac{b_{22}}{b_{12}}>\frac{\lambda_{2}^{(1 / k)}-1}{\lambda_{1}^{(1 / k)}-1}
$$

and that coexistence in model 2 requires

$$
\frac{a_{22}}{a_{12}}>\frac{\lambda_{2}^{(1 / k)}-1}{\lambda_{1}^{(1 / k)}-1}
$$

\section{Stability}

A complete stability analysis of the nontrivial equilibria we have found seems almost impossible in general and would clearly depend on specific functional forms. Even for specific functional forms, the algebra required to determine stability is likely to be impossibly messy. We give three different arguments supporting stability when $k<$ 1 and when either (31) or (35) are met.

First, for both models we note that, if each host-parasitoid pair is stable in isolation, then we can use bifurcation techniques (e.g., Iooss and Joseph 1980; Hastings 1982) as explained in the appendix to prove that the full system will have a stable equilibrium near the equilibrium with the less fecund host absent, for values of $a_{22}$ or $b_{22}$ just larger than those permitting the host with lower fecundity to enter.

A second case where perturbation theory can be used to show stability, which also helps illustrate the mechanism promoting coexistence, begins with the extreme parameter values having $b_{i j}$ or $a_{i j} 0$ for $i \neq j$ and 1 for $i=j$. Then the two species model breaks up into two isolated systems. If these systems are both stable, the results of Karlin and McGregor (1972) can be used to show that the full system will have a stable equilibrium for values of $b_{i j}$ or $a_{i j}$ close to the extreme case.

The final approach we offer for examining stability is to look at simulations of the full system. We have done this for the negative binomial case, and if the condition for stability of a single host-parasitoid pair $(k<1)$ is met together with our conditions for coexistence, the full system always appears to produce stable coexistence.

\section{Discussion}

Our models show that the type of learning of host location kairomones that has recently been demonstrated by parasitoid physiologists is capable of contributing to the co- 
existence of two species that are regulated by a single parasitoid. Coexistence requires that the strength of host fidelity is sufficient to provide the species with lower than maximum fecundity a means of increasing when rare. The result occurs whether parasitoids search simultaneously for all species with differing efficiencies, or whether search is mutually exclusive. Our models consider a parasitoid attacking only two species of hosts, but we believe that similar results will be found for species with larger host ranges and that this form of learning will contribute to the stability of more complex webs containing many species of hosts and parasitoids.

We have concentrated on behavioral mechanisms since it was these that motivated our modeling. However, we note that our models may provide a phenomenological description of other types of parasitoid behavior. Consider a population of parasitoids attacking two host species that are partially spatially segregated in the environment. On emergence, the parasitoid begins searching in its immediate vicinity and, hence, will be more likely to encounter hosts of the same species. This process is mimicked by our models in which the relative probability a parasitoid individual attacks a particular host species is determined by its natal host.

We finish by mentioning some simplifications of our model and how it might be extended. Throughout we have assumed a linear functional response, effectively that the parasitoid has unlimited eggs and a very short handling time. Violations of these assumptions will affect both the ability of the parasitoid to regulate its hosts and the criterion for coexistence (Hassell and May 1973). We have also restricted our analysis to a simple difference equation framework and have not tried to model explicitly the changing state of the parasitoid over its life span. Models in which the dynamic effects of parasitoid decision making have been studied using explicitly varying states have been developed for host feeding (whether to lay an egg or feed from a host), clutch size, and patch use (Murdoch et al. 1997) and could be extended to study the type of host range problem discussed here.

Finally, we have assumed that the parasitoid's behavior is fixed and represents a past adaptation to improve host searching efficiency. An interesting extension would be to model optimal levels of host fidelity under different ecological circumstances, and to explore the interplay between ecological and evolutionary stability.

\section{Acknowledgments}

This research was supported in part by National Science Foundation grant DEB 96-28937 to A.H. A.H. also thanks the visitors' program of the Natural Environment Research Council Centre for Population Biology for financial support. We thank D. Strong for helpful comments.

\section{APPENDIX}

\section{Bifurcation Analysis}

We will present a bifurcation argument that shows that there will be a stable equilibrium with both host species present for parameter values that correspond to an equilibrium with both hosts present but are sufficiently close to the parameter values at which the conditions (23) or (31) change from not being satisfied to being satisfied. We present the details for model 1 ; model 2 is similar. The argument depends on first showing that the boundary equilibrium with $\hat{H}_{1}=0$, but $\hat{P}_{1}>0, \hat{H}_{2}>0$, and $\hat{P}_{2}>0$ changes stability when (23) changes from not satisfied to satisfied. (We use an alternate notation for equilibria to distinguish the boundary equilibrium from the interior equilibrium.) The second step is to show that the interior equilibrium exists only when the boundary equilibrium is unstable.

The Jacobian for the system (5) and (6), evaluated at the equilibrium with $\hat{H}_{1}=0$ (with the variables arranged in the order $H_{1}, P_{1}, H_{2}, P_{2}$ ) is

$$
\left(\begin{array}{cccc}
\lambda_{1} \mathrm{f}\left(\hat{P}_{1}\right) & 0 & 0 & 0 \\
b_{11}\left[1-\mathrm{f}\left(\hat{P}_{1}\right)\right] & 0 & b_{12}\left[1-\mathrm{f}\left(\hat{P}_{2}\right)\right] & -b_{12} \hat{H}_{2} \frac{\partial \mathrm{f}}{\partial P_{2}} \\
0 & 0 & \lambda_{2} \mathrm{f}\left(\hat{P}_{2}\right) & \lambda_{2} \hat{H}_{2} \frac{\partial \mathrm{f}}{\partial P_{2}} \\
b_{21}\left[1-\mathrm{f}\left(\hat{P}_{1}\right)\right] & 0 & b_{22}\left[1-\mathrm{f}\left(\hat{P}_{2}\right)\right] & -b_{22} \hat{H}_{2} \frac{\partial \mathrm{f}}{\partial P_{2}}
\end{array}\right) .
$$

Because of the conveniently placed zeroes in this Jacobian, the eigenvalues are easily seen to be $\lambda_{1} \mathrm{f}\left(\hat{P}_{1}\right), 0$, and the eigenvalues of the subsystem consisting only of $\mathrm{H}_{2}$ and $P_{2}$,

$$
\left(\begin{array}{cc}
\lambda_{2} \mathrm{f}\left(\hat{P}_{2}\right) & \lambda_{2} \hat{H}_{2} \frac{\partial \mathrm{f}}{\partial P_{2}} \\
b_{22}\left[1-\mathrm{f}\left(\hat{P}_{2}\right)\right] & -b_{22} \hat{H}_{2} \frac{\partial \mathrm{f}}{\partial P_{2}}
\end{array}\right),
$$

corresponding to the lower right-hand corner of (A1).

We have assumed that the subsystem is stable when $H_{1}=0$. Thus, we see that when the full system has no equilibrium with $H_{1}>0$, which implies that $\lambda_{1} \mathrm{f}\left(\hat{P}_{1}\right)<1$, 
the full system has a stable equilibrium with $H_{1}=0$. Conversely, when the full system has an equilibrium with $H_{1}>0$, which implies that $\lambda_{1} \mathrm{f}\left(\hat{P}_{1}\right)>1$, the equilibrium for the full system with $H_{1}=0$ is unstable. Standard arguments from bifurcation theory (Iooss and Joseph 1980; Hastings 1982) imply that close to the bifurcation point (where the boundary equilibrium with $H_{1}=0$ loses stability) the interior equilibrium is stable since it occurs for those parameter values where the boundary equilibrium is unstable. A similar argument works for model 2.

\section{Literature Cited}

Chesson, P. L., and W. W. Murdoch. 1986. Aggregation of risk: relationships among host-parasitoid models. American Naturalist 127:696-715.

Corbett, S. A. 1985. Insect chemosensory response: a chemical legacy hypothesis. Ecological Entomology 10: 143-153.

Cortesero, A. M., and J. P. Monge. 1994. Influence of preemergence experience on response to host and host plant odours in the larval parasitoid Eupelmus vuilleti. Entomologia Experimentalis et Applicata 72:281-288.

Godfray, H. C. J. 1994. Parasitoids: behavioral and evolutionary ecology. Princeton University Press, Princeton, N.J.

Godfray, H. C. J., and C. B. Müller. 1998. Host-parasitoid dynamics. In J. P. Dempster and I. Maclean, eds. Insect population dynamics. Academic Press, London.

Hassell, M. P. 1978. The dynamics of arthropod predatorprey systems. Princeton University Press, Princeton, N.J.

- 1998. The dynamics of insect-parasitoid interactions. Oxford University Press, Oxford.

Hassell, M. P., and R. M. May. 1973. Stability in insect host-parasite models. Journal of Animal Ecology 42: 693-726.

Hastings, A. 1982. Unexpected behavior in two-locus genetic systems: an analysis of marginal underdominance at a stable equilibrium. Genetics 102:129-138.

Holt, R. D., and J. H. Lawton. 1993. Apparent competition and enemy-free space in insect host-parasitoid communities. American Naturalist 142:623-645.

Iooss, G., and D. D. Joseph. 1980. Elementary stability and bifurcation theory. Springer, New York.

Karlin, S., and J. McGregor. 1972. Polymorphisms for genetic and ecological systems with weak coupling. Theoretical Population Biology 3:210-238.

Kester, K. M., and P. Barbosa. 1991. Post-emergence learning in the insect parasitoid, Cotesia congregata (Say). Journal of Insect Behavior 4:727-742.
May, R. M. 1978. Host-parasitoid systems in patchy environments: a phenomenological model. Journal of Animal Ecology 47:833-843.

Memmott, J., H. C. J. Godfray, and I. D. Gauld. 1994. The structure of a tropical host-parasitoid community. Journal of Animal Ecology 63:521-540.

Mills, N. J., and W. M. Getz. 1996. Modelling the biological control of insect pests: a review of host-parasitoid models. Ecological Modelling 92:121-143.

Murdoch, W. W., and C. J. Briggs. 1996. Theory for biological control: recent developments. Ecology 77: 2001-2013.

Murdoch, W. W., and A. Oaten. 1975. Predation and population stability. Theoretical Population Biology 12: 263-285.

Murdoch, W. W., C. J. Briggs, and R. M. Nisbet. 1997. Dynamical effects of host size and parasitoid state-dependent attacks by parasitoids. Journal of Animal Ecology 66:542-556.

Nicholson, A. J., and V. A. Bailey. 1935. The balance of animal populations. I. Proceedings of the Zoological Society of London 3:551-598.

Nisbet, R. M., and W. S. C. Gurney. 1983. The systematic formulation of population models for insects with dynamically varying instar duration. Theoretical Population Biology 23:114-135.

Thorpe, W. H., and F. G. W. Jones. 1937. Olfactory conditioning in a parasitic insect and its relation to the problem of host selection. Proceedings of the Royal Entomological Society of London A 124:56-81.

Tilman, D. 1982. Resource competition and community structure. Princeton University Press, Princeton, N.J.

Turlings, T. C. J., F. L. Wackers, L. E. M. Vet, W. J. Lewis, and J. H. Tumlinson. 1992. Learning of host-location cues by hymenopterous parasitoids. Pages 51-78 in A. C. Lewis and D. R. Papaj, eds. Insect learning: ecological and evolutionary perspectives. Chapman \& Hall, New York.

Vet, L. E. M. 1983. Host-habitat location through olfactory cues by Leptopilina clavipes (Hartig) (Hym: Eucoilidae), a parasitoid of fungivorous Drosophila: the influence of conditioning. Netherlands Journal of Zoology 33: 225-248.

Vet, L. E. M., and A. W. Groenewold. 1990. Semiochemicals and learning in parasitoids. Journal of Chemical Ecology 16:3119-3135. 